\title{
Application of Noddings' Care Ethics in the Communication of Chinese Amputation Patients and Related Clinical Teaching
}

\author{
Hongtao Xiong*, Zhouyong Tan, Xiaokuan Fu, Xuexian Wen \\ Department of Hand and Microvascular Surgery, Shenzhen People’s Hospital (The Second Clinical Medical College, Jinan Uni- \\ versity; The First Affiliated Hospital, Southern University of Science and Technology), Shenzhen 518020, Guangdong, China.
}

\begin{abstract}
How to cite this paper: Hongtao Xiong, Zhouyong Tan, Xiaokuan Fu, Xuexian Wen. (2021). Application of Noddings' Care Ethics in the Communication of Chinese Amputation Patients and Related Clinical Teaching. The Educational Review, USA, 5(7), 191-195.

DOI: 10.26855/er.2021.07.001
\end{abstract}

Received: June 18, 2021

Accepted: July 10, 2021

Published: July 14, 2021

Corresponding author: Hongtao Xiong, Department of Hand and Microvascular Surgery, Shenzhen People's Hospital (The Second Clinical Medical College, Jinan University; The First Affiliated Hospital, Southern University of Science and Technology), Shenzhen 518020, Guangdong, China.

Email: xionghongtaosz@126.com

\begin{abstract}
The ethics of Noddings' care is an ethical theory which discusses the responsibility, emotion, relationship and mutual care between people, which has strong applicability in the relationship between doctors and patients. Amputation has the function of disability and is also a great blow to the patients' psychology. Under the influence of traditional culture, Chinese people have a lower reception degree of this operation, and need to give more professional care. Besides the indication and necessity of amputation, the medical side should also consider the acceptance of patients and their families. This is related to the age and occupation, cultural customs, ideological cognition, economic situation of the patients, and not only the medical technology. In clinical work, we should fully recognize the necessity and limitation of this surgical treatment method, and pay attention to the advantages and disadvantages of amputation. This paper summarizes the effect of doctor-patient communication and clinical teaching of 22 amputated patients, discusses the use of Noddings' care ethics knowledge to realize the good communication between doctors and patients, and at the same time, the specific application methods of Noddings' care ethics are transferred to lower doctors, and finally achieve the equal and reciprocal interpersonal relationship among doctors, patients and students.
\end{abstract}

\section{Keywords}

Noddings' caring ethics, Amputation, Doctor-patient communication, Clinical medicine teaching

\section{Introduction}

According to Zhang (2018) and Wang (2019), medical humanities is a universal value and social movement, which is suitable for the social development and change. The report (2010) of China medical patient relationship survey by the statistics information center of the Ministry of Health pointed out that the service attitude of Chinese medical parties is the disaster area of patients' complaints. Meanwhile, many studies believe that Noddings' care ethics can provide inspiration and feasible ways for building harmonious doctor-patient relationship, and also an important part of clinical medical humanities education (Han et al., 2014; Peng et al., 2017; Balmer DF et al., 2016).

This paper summarizes 22 amputation patients (22), aged 57-78, 16 males and 5 females, from January 2017 to December 2019. The knowledge of Noddings' care ethics was used to communicate between doctors and patients, and no 
one patient complained; at the same time, 30 medical students, including residents and interns were completed clinical learning with highly satisfying experience. All medical students participated in the communication process between doctors and patients. The satisfaction rate of medical students was $90 \%$ according to the teaching results.

\section{Basic content of Noddings' care ethics}

Nell Noddings is a representative of modern ethics of care. Noddings thinks that care includes two meanings: first, if an individual is concerned about something or feels that he should do something about it, he is caring about it; second, if the individual has expectations and concerns about someone, it means that he is caring for the person. Therefore, it can be seen that care is not only about concern, but also for something or someone, protecting their interests and promoting their development. At the same time, care is the interaction and reciprocity between the concerned and the concerned. The concerned people should respond to the actions of the concerned people while caring for the concerned through their own actions.

Noddings believes that when it comes to the emotional feeling of love, it is just because of the instinctive impulse of the carer, which is similar to the "compassion" in Chinese traditional culture; on the contrary, only under the encouragement of certain ethical ideal can the concern response to this need be produced, such care is ethical concern. Although natural care can accumulate the memory of care and being cared, and then strengthen it as ethical care, natural care is the basis of ethical care, Noddings pointed out (2002). Because of the limited natural care, Noddings believes that the scope of interpersonal care can be expanded by developing ethical care. Doctors should develop this kind of ethical care to patients and teachers to students (Guo \& Qian, 2020).

\section{Comprehensive medical risk of amputation}

Generally, patients will ask to keep as many parts as possible, which appear normal, and only the necrotic part is removed, but this may cause the wound to heal, increase the number of operations, and thus bring more risks. The incidence rate of hypertension, diabetes, coronary heart disease and arteriosclerosis in elderly patients in China is high. Every time of operation, myocardial infarction, cardiac arrest, stroke and even vegetative state may occur. Due to the lack of follow-up treatment and nursing rehabilitation funds, once stroke, vegetative state or death occurs, following the previous study (Xie et al., 2014), the family members of the patients are unable to bear and refuse to accept it. For the purpose of anger relief and compensation, medical trouble may appear. Even if it is a complete amputation operation, for patients with high risk of stroke, the possibility of more serious stroke, becoming a vegetative state or dying is greater in the perioperative period. At the same time, the limb defect after operation will be difficult for the patients to accept (Song et al, 2015). The risk of choosing safe plane and site amputation is small, and the prosthesis can also achieve better results.

Therefore, the application of Western medicine in China should take into account the cultural tradition, social development, the cognition, knowledge level and economic situation of the patients. It is wrong to see a doctor only and not to see people. The first thing to see is the patient's complication, the second is to see the cognitive and cultural background of the patient, which requires full communication and deep communication. Chinese Western medical students should learn and understand this key point from beginning to end, flexibly use the knowledge learned, avoid falling into the misunderstanding that only looking at legs and not looking at other parts, only seeing doctors and not seeing people, and fully respecting the patients' right to know and choose.

\section{Application of Noddings' care ethics in amputation}

Noddings believes that concern is the basis of ethics, and the relationship is the ontological basis of concern. The two sides of the relationship, the concerned and the concerned, are mutually beneficial in the relationship of concern, but they are not equal. The concerned need to show the focus and motivation shift in the care, while the concerned need to respond in some way so that the relationship of concern can be completed. The theory of caring of Noddings includes three elements: the good motivation of the caretaker and the devotion of the whole body; the needs and reactions expressed by the object of concern in a certain way; the consciousness, recognition and response of the care object to the care behavior. It is the "completion of each other" between the care and the object concerned (1988).

As one of the concerned parties in the relationship of concern, clinicians should fully communicate with patients in terms of symptoms, changes of general condition, wound healing, psychological changes and social adaptability, etc., listen to the discomfort of the related party and the pain of the heart, and give clinical treatment and psychological support, to promote the physical and mental recovery of patients and the improvement of social adaptation. In the process of getting patients to get good return from physical, psychological and social aspects, the medical side can see good 
treatment effect, and the psychological pressure during the whole treatment stage is fully released, and the sense of achievement and satisfaction is obtained. The relationship of care is finally completed. Doctors as concerned, the focus of different stages is different, before the operation, focus on the patient's physical condition, to ensure the perioperative patients' life safety; the operation process should be focused on to ensure the smooth recovery of the wound; the postoperative focus is on complications and wound rehabilitation, as well as the follow-up mental and social adaptability.

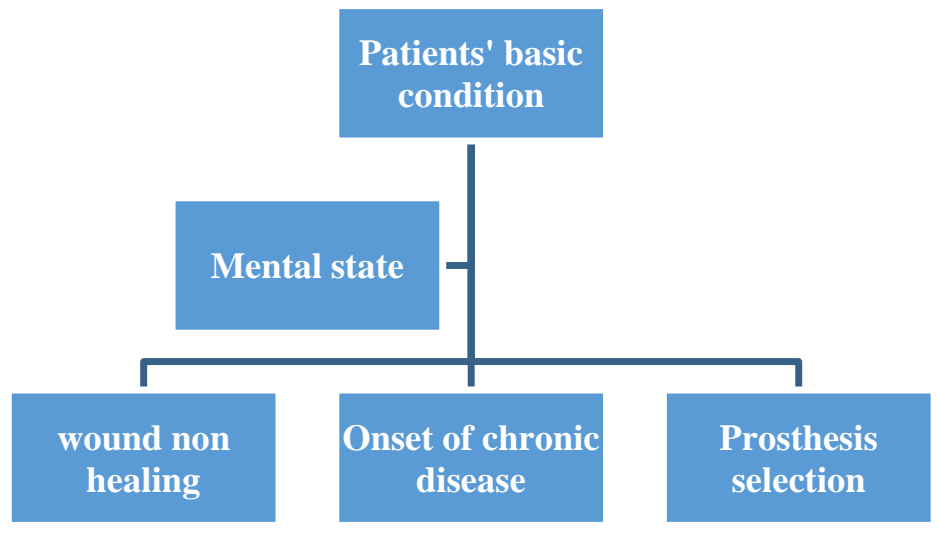

Figure 1. Patients with focus.

As shown in Figure 1, the patient's general condition should be gradually improved, and the symptoms of toxic substance absorption caused by the necrosis of the original limbs should be significantly improved. Meanwhile, the patient's mental state improved with the treatment. Because most of the patients are elderly, and complicated with chronic diseases, the wound healing ability is poor, we should pay attention to the infection, nonunion and other problems caused by congestion in the wound, early detection and targeted treatment can avoid more serious complications. After amputation, when the limb deformity is confirmed, the patients are more or less depressed. To provide patients with new progress and achievements in prosthetic research and application, to point out the direction of limb functional reconstruction after discharge, and to help patients regain their confidence in life.

In the specific methods of implementing caring behavior, clinicians should pay attention to the implementation of four links: example, dialogue, practice and recognition (Chen, 2010), take patient satisfaction as the purpose, and promote rehabilitation as the goal. According to the physiological, psychological and social adaptation needs of amputees, we should carry out effective dialogue and care measures in a timely and appropriate manner; through repeated practice, it is finally recognized by both doctors and patients to further strengthen the caring relationship between the caregiver and the cared.

\section{The value of Noddings' caring ethics in medical humanities education}

According to Noddings, caring is a kind of relational behavior, and its maintenance depends on people's love emotion (Noddings et al., 1996). The specific expression of this love emotion in the practice of medical humanities education is to enter the meaning world of medical students and realize the meaning care for medical students. The main object of medical humanities education is people, namely medical students. Under the baptism of medical humanistic knowledge, guiding medical students to realize the sacred meaning of medical profession, so that they can enjoy the inherent dignity and happiness brought by medical profession, is the proper meaning of ideal medical humanistic education (Shi, 2014). Only after the superior doctors fully explain the etiology and pathology of foot necrosis to the patients and medical students, can the medical students understand that amputation is the best choice, and they can get a better quality of life after amputation. On the contrary, the perioperative risk of amputation is the medical problem that needs to be paid attention to.

Under the influence of traditional culture and social culture, patients and their families after amputation are often depressed because of limb deformity, and will not be happy because of the benefits of amputation. When junior doctors or interns see this situation, they may have the wrong understanding of treatment choice or treatment failure, leading to their own emotional depression. As a superior physician, he should have sufficient confidence and full understanding, not only to recognize the disability effect of surgery, but also to fully realize the benefits and effective therapeutic effect 
of amputation on patients; we should not only recognize that this process is the inevitability of the growth of surgeons, but also take into account the fragile psychology when we first enter the clinic, so as to provide psychological counseling for the younger generation and increase the confidence of being a doctor (Guan, 2015).

\section{Discussion}

With the development of modern China's social economy, more and more patients with hypertension, hyperglycemia and hyperlipidemia are suffering from "Three High" diseases in China. The common result is the rapid increase of atherosclerosis. It is not uncommon for lower extremity arteriosclerosis obliterans or diabetic foot to cause foot necrosis and eventually to undergo amputation. As for amputation caused by injury or terminal malignant tumor, the patients' acceptance is slightly higher, but resistance psychology is still very heavy. Influenced by Chinese traditional culture, "Body, skin and hair were from parents, and cannot be hurt", Chinese people accept amputation far lower than western. Such situations are often met in clinical practice, which is a problem that must be faced in clinical teaching. Amputation, as a final treatment, is a measure that has to be taken to save the life of the patient in the case of failure of limb or limb salvage treatment. In the process of communication with patients, in addition to explaining the indications, necessity and risks of surgery, it is necessary to fully understand the patients' thoughts, including the psychological and ideological understanding of the patients, the acceptance of amputation by patients and their families, the psychological endurance of patients, and even the life conditions after amputation, etc., which are more complex, such as improper treatment, it is easy to cause medical disputes. In this regard, Noddings' caring ethics can improve the doctor-patient relationship, and it is also an important part of medical education (Balmer et al., 2016).

\section{Conclusions}

During the follow-up treatment for the patients after the operation, the superior teachers communicated with the patients and conducted psychological counseling for the patients. At the same time, they also did amputation, which is the positive application of Noddings' educational concept in medical practice education, and the concrete embodiment of medical humanities education in the classroom, it is one of the ultimate goals of medical humanities education (Fujino, 2016). In the process of interaction and communication with doctors, patients and students, the higher-level doctors play an effective role in professional technology teaching, humanistic care for patients, medical ethics teaching of amputation, reexamination of amputation and comprehensive quality training of lower-level doctors, so as to achieve the good effect of "harmony between doctors and patients" and "mutual benefit between teaching and learning".

\section{References}

Balmer, D. F., Hirsh, D. A., Monie, D., et al. (2016). Caring to care: Applying Noddings’ Philosophy to Medical Education. Academic Medicine, 91(12): 1618-1621. https://doi:10.1097/ACM.0000000000001207.

Chen, S. K. (2010). The humanistic value of Noddings’ caring ethics. Zhong Guo Xue Shu Yue Kan, 2010(4): 19-21.

Fujino, A. (2015). Medical Humanities - the Historical Significance and Mission in Medical Education. Journal of UOEH, 1, 37(4): 273-91. https://doi: 10.7888/juoeh.37.273.

Guan, Y. Y., Wang, J. F., and Shen, H. B. (2014). Discussion on the cultivation of medical humanistic practice ability of medical students. Yi Xue Yu Zhe Xue(a), (9): 88-90.

Guo, S. L., Qian. Z. G. (2011). The path of cross-cultural teaching to improve the humanistic quality of medical students. Chinese Journal of Bengbu Medical College, 36(1): 93-95. https://doi: 10.3969/j.issn.1000-2200.2011.01.036.

Han. P., Chen. X. Y., Zhang. T. S., et al. (2014). Qualitative research on the application of medical humanistic care in the improvement of doctor-patient relationship. Zhong Guo Yi Xue Lun Li Xue, 27(2): 284-287.

Noddings, N. (1998). An ethic of caring and its implications for instructional arrangements. America Journal of Education, 96: 215-230.

Noddings, N. (2002). Starting at home: caring and social policy (pp. 116-118). Berkeley: University of California Press.

Noddings, N., Gordon, S., and Benner, P. (1996). The caring professional. In: Caregiving: Readings in Knowledge, Practice, Ethics and Politics. Philadelphia, Pa (pp. 160-172). University of Pennsylvania Press.

Peng, J., Zhang, W. L., Zhang. J. Z., et al. (2017). The Enlightenment of Noddings' caring ethics on the construction of doctor-patient relationship in China. Yi Xue Yu Zhe Xue, 38(5): 60-62. https://doi: 10.12014/j.issn.1002-0772.2017.03a.14.

Shi, T. J. (2014). Caring for meaning: Noddings' caring ethics and its value proposition of medical humanities education. Zhong Guo Ji Chu Yi Xue Jiao Yu, 2014(16): 980-983. https://doi: 10.13754/j.issn2095-1450.2014.11.34. 
Song. F. H., Li, J. L., and Liu, L. (2015). Management and prevention of complications of inferior genicular artery angioplasty in patients with diabetic lower genicular artery occlusion. Zhong Guo Lin Chuang Wu Zhen Wu Zhi, 8(3): 59-61. https://doi:10.3969/j.issn.1002-3429.2015.03.022.

Statistical Information Center of Chinese Ministry of Health. (2010). Investigation of doctor patient relationship in China: Special Research Report of the fourth national health service survey. (pp. 273-279). Beijing: Union Medical University Press.

Wang, W. H., Wu, S. Y., and Ke, M. (2019). On medical humanities practice ability and its cultivation. Yi Xue Yu Zhe Xue, 39(10a): 70-73. https://doi: 10.12014/j.issn.1002-0772.2018.10a.16.

Xie, Y. L., Cai, L., Wang, W. D., et al. (2014). Reflections on medical ethics in the treatment of patients with limb bone tumors. Zhong Guo Yi Xue Lun Li Xue, 27 (4): 564-566.

Zhang, D. Q. (2018). Medical humanities, medical tradition and social culture. Zhong Guo Yi Xue Lun Li Xue, 31(4), 511-512. https://doi: 10.12026/j.issn.1001-8565,2018.04.23. 